\title{
Astrocytes: everything but the glue
}

\author{
Oscar Gonzalez-Perez ${ }^{1}$, Veronica Lopez-Virgen ${ }^{1}$, Alfredo Quiñones-Hinojosa ${ }^{2}$ \\ ${ }^{1}$ Laboratory of Neuroscience, School of Psychology, University of Colima, Colima, Col 28040, Mexico. \\ ${ }^{2}$ Department of Neurosurgery, Johns Hopkins School of Medicine, Baltimore, MD 21201, USA.
}

The current knowledge in neuroscience indicates that the neural tissue has two major cell populations: neurons and glia (term derived from the Greek word for glue). Neuronal population is characterized by the capacity to produce action potentials, whereas glial cells are typically identified as the subordinate cell population of neurons. Till date, this point of view has changed dramatically, and growing evidence indicates that glial cells play a crucial role in normal mental functions and the pathogenesis of neurological diseases. Classically, glial cells include four major populations clearly discernible in the adult's brain: astrocytes, oligodendrocytes, microglia cells and NG2 glia. Astrocytes, also referred as to astroglia, are by far the most abundant cell lineage in the adult's brain and display a very diverse morphology, which varies during neural development and adulthood [Figure 1]. These cells are in close contact with several tissue components of the brain parenchyma including neurons, vasculature, extracellular matrix and other glial populations. Hence, the number and strategic position of astrocytes provide them with exceptional capacity for modulating multiple functions in the neural tissue.

During neural development, the origin of astrocyte varies according to the anatomical region, but the most accepted viewpoint suggests that astrocytic cells originate from radial glial cells (in the ventricular zone) and some progenitor cells located in the subventricular zone (SVZ). Therein, astrocyte lineage is directed by the expression of notch receptors and their ligands (delta and serrate-jagged) that efficiently repress neuronal fate and promotes gliogenesis. ${ }^{[1]}$ At this developmental stage, astrocytes are crucial for the synapse formation by improving the neuron's ability to receive synaptic contacts, which consequently initiate neuronal

\begin{tabular}{|l|l|}
\hline \multicolumn{2}{|c|}{ Access this article online } \\
\hline Quick Response Code: & \\
\hline & Website: \\
\hline & www.nnjournal.net \\
\cline { 2 - 2 } & DOI: \\
\hline $\mathbf{D}$ & $10.4103 / 2347-8659.153979$ \\
\hline
\end{tabular}

communication. ${ }^{[2]}$ Postnatal synapse refinement is also associated with astrocyte maturation suggesting that the astroglia is an important modulator of neuronal plasticity. ${ }^{[3]}$

Astrocytes are ubiquitous brain cells that outnumber neuronal population in the proportion of 10:1 and comprise approximately $50 \%$ of the neural tissue. Before 1980 's, the role of astrocytes was basically associated with supporting and nourishing neurons (primarily considered as a "real" functional cells). However, current evidence indicates that this perspective is quite limited and incorrect. Today, it is well accepted that astrocytes have a more major role in neurological functioning, neural homeostasis and pathogenesis than previously thought. ${ }^{[4]}$

Astroglia is the main source of glycogen that delivers energy to the brain interstitial tissue and nourishes neurons by providing lactate. ${ }^{[5]}$ Other astrocytic functions include: the uptake or release of neurotransmitters, ${ }^{[6]}$ modulation of synaptic plasticity and neuronal transmission, ${ }^{[3]}$ regulation of interstitial ions, ${ }^{[7]}$ cerebral regulation of blood flow and blood-brain barrier, ${ }^{[8]}$ myelin regulation, ${ }^{[9]}$ tissue repair, ${ }^{[10]}$ neuroprotection ${ }^{[11]}$ and drug metabolism. ${ }^{[12]}$

In addition, astrocytes have astonishing enzymatic machinery that includes cyclooxygenase, lipoxygenase and cytochrome P450 epoxygenase pathways. ${ }^{[12]}$ Remarkably, these enzymes allow astrocytes to depurate several drugs and alcohol, a property that was previously thought to be exclusive of hepatocytes (liver cells). Astroglia can also produce metabolic intermediates of polyunsaturated fatty acid arachidonic acid and epoxide metabolites that may help protect against stroke. Nevertheless, some gene mutations on astrocytes produce defective proteins that upon accumulation may lead to neurodegenerative diseases. Besides, the phospholipase-C (PLC) enzymatic family and the phosphoinositide signaling system are essential for molecule transduction from the cell membrane to the nucleus. ${ }^{[12]}$ This system appears to be involved

Corresponding Author: Dr. Oscar Gonzalez-Perez, Laboratory of Neuroscience, School of Psychology, University of Colima, Av. Universidad 333, Colima, Col 28040, Mexico. E-mail: osglez@ucol.mx 


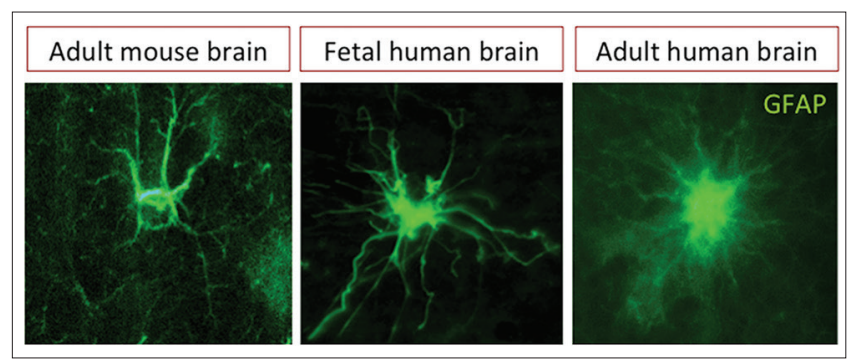

Figure 1: Immunofluorescence images of parenchymal astrocytes. Astrocytic processes in the adult human brain are more profuse and thinner than in the rodent or fetal brain, glial fibrillary acidic protein

in a number of neuronal and glial functions. Up- or down-regulation of different phosphoinositide-specific PLC isoforms (PI) can drive astrocyte reactivity associated with brain inflammation and tumor progression. ${ }^{[13]}$ PI-PLC $\beta 1$, PI-PLC $\beta 4$ and PI-PLC 1 have been found in reactive astrocytes, whereas PI-PLC $\beta 3$, PI-PLC 2, PI-PLC 2, PI-PLC PI-PLC have been identified in brain tumor cells (astrocytoma). However, the clinical significance of these findings remains to be clarified. ${ }^{[14]}$ In this regard, the expression of nicotinamide adenine dinucleotide phosphate ${ }^{+}$-dependent isocitrate dehydrogenase-1 gene and epidermal growth factor (EGF) appear to distinguish reactive astrocytes from glioma, but further validation is required to confirm these findings. ${ }^{[15]}$

Neurotropic viruses are infectious particles that can preferentially infect neural cells by evading the brain immune response and infecting astroglia. Such viral infection in astrocytes alters their functions and lead to serious neural damages with severe neurological complications. In response to the viral infection, current evidence indicates that activated astrocytes promote innate immune response through the expression of toll-like receptors and secretion of cytokines, that is, astrocytes appear to direct immune response and neuroinflammation. ${ }^{[13]}$

Astrocytes contain inward-rectifier $\mathrm{K}^{+}$(Kir) channels that efficiently maintain potassium $\left(\mathrm{K}^{+}\right)$homeostasis during neuronal activities. ${ }^{[16]}$ Changes in the expression and functioning of astrocytic Kir channels modify $\mathrm{K}^{+}$spatial buffering. The reduced Kir4.1 channel expression or Kir4.1 channel misallocations promote astrocyte depolarization, which decreases their ability to clear extracellular glutamate. This mechanism is responsible for the neural damage in epilepsy and ischemia. Hence, novel therapeutic approaches have been suggested to involve the regulation of $\mathrm{K}^{+}$spatial buffering by astrocytes.

Since astrocytes are responsible for the optimal ionic homeostasis and neurotransmission balance, astrocytic atrophy has been associated with early alterations of the neuronal transmission and synaptic networks observed in several neurodegenerative diseases including Alzheimer's disease (AD). In later alterations of $\mathrm{AD}$, senile plaques trigger glial reactivity that, in turn, advances neuronal death and cognitive deficit. Recently, it has been demonstrated that reactive astrocytes produce the inhibitory gliotransmitter GABA, which in consequence reduces the synaptic plasticity and the performance of learning and memory in a mouse model of AD. ${ }^{[17]}$ Nevertheless, the role of astrocytes in the pathogenesis or progression of neurodegenerative diseases is not completely understood.

A subpopulation of astrocytes can also function as neural stem cells in the adult's brain. Neural stem cells reside in the ventricular-SVZ (V-SVZ), a cell niche lining the lateral wall of the lateral ventricles. ${ }^{[18]}$ Therein a subpopulation of astrocytes functions as bona fide neural stem cells that generate a number of neuroblasts that rostrally migrate and differentiate into interneurons at the olfactory bulb. ${ }^{[18]} \mathrm{V}-\mathrm{SVZ}$ astrocyte progenitors can also promote the genesis of oligodendrocytes that populate white matter tracts of corpus callosum and striatum. ${ }^{[19]}$ Interestingly, the intraventricular administration of EGF increases dramatically the production of V-SVZ oligodendrocytes and NG2-platelet-derived growth factor receptor- $\alpha$ expressing cells, which temporarily disrupt the neighboring white matter. ${ }^{[19]}$ Oligodendrocyte dysfunction has been found across most psychiatric conditions, including mood disorders. ${ }^{[20]}$ However, the role of V-SVZ oligodendrogenesis in the pathophysiology of neuropsychiatric disorders remains to be elucidated.

In the adult hippocampus, another subpopulation of astrocytes gives rise to new neurons that populate the granular layer of the dentate gyrus. ${ }^{[21]}$ These multipotent astrocytes help control several behavioral tasks, such as episodic-like and spatial memory, object recognition tasks and cognitive performance. ${ }^{[22]}$ Interestingly, morphological changes and local distribution of astrocytes are dependent on aging and enriched environments. Therefore, astrocytic neural stem cells in the V-SVZ and dentate gyrus help preserve cellular homeostasis and exert modulatory roles on neural circuitry in the adult's brain.

Astrocytes not only preserve the oligodendrocyte population, ${ }^{[23]}$ but also modulate the myelination process of oligodendrocytes. ${ }^{[2]}$ Some mutations in astrocytes are associated with leukodystrophies, a group of neurological disorders characterized by imperfect myelin ensheathing. Therefore, although the origin of megalencephalic leukoencephalopathy was initially related to the malfunction of oligodendrocyte, emerging evidence indicates that the pathophysiology is associated with astrocytic dysfunction. 
In summary, astrocytes are vital cell mediators for the cerebral homeostasis and play an important role in the pathophysiology and progression of a number of brain disorders. Every day, new evidence emerges to reveal that the glial cells are much more than supporting and nourishing cells in the brain. Thus, astrocytes are everything plus the glue.

\section{ACKNOWLEDGMENTS}

The National Institute of Health and the National Institute of Neurological Disorders and Stroke (NIH/ NINDS; R01-NS070024), Consejo Nacional de Ciencia y Tecnologia (CONACyT; INFR2014-224359). Programa de Desarrollo del Personal Docente (PRODEP-Redes 2014).

\section{REFERENCES}

1. Nye JS. The Notch proteins. Curr Biol 1999;9:R118.

2. Barker AJ, Ullian EM. New roles for astrocytes in developing synaptic circuits. Commun Integr Biol 2008;1:207-11.

3. Ota Y, Zanetti AT, Hallock RM. The role of astrocytes in the regulation of synaptic plasticity and memory formation. Neural Plast 2013;2013:185463.

4. Pekny M, Pekna M. Astrocyte reactivity and reactive astrogliosis: costs and Benefits. Physiol Rev 2014;94:1077-98.

5. Barros LF. Metabolic signaling by lactate in the brain. Trends Neurosci 2013;36:396-404

6. Halassa MM, Haydon PG. Integrated brain circuits: astrocytic networks modulate neuronal activity and behavior. Annu Rev Physiol 2010;72:335-55.

7. Seifert G, Steinhäuser C. Neuron-astrocyte signaling and epilepsy. Exp Neurol 2013;244:4-10.

8. Cabezas R, Avila M, Gonzalez J, El-Bachá RS, Báez E, García-Segura LM, Jurado Coronel JC, Capani F, Cardona-Gomez GP, Barreto GE. Astrocytic modulation of blood brain barrier: perspectives on Parkinson's disease. Front Cell Neurosci 2014;8:211.

9. Lundgaard I, Osório MJ, Kress BT, Sanggaard S, Nedergaard M. White matter astrocytes in health and disease. Neuroscience 2014;276:161-73.

10. Kang K, Lee SW, Han JE, Choi JW, Song MR. The complex morphology of reactive astrocytes controlled by fibroblast growth factor signaling. Glia 2014;62:1328-44.

11. Yao PS, Kang DZ, Lin RY, Ye B, Wang W, Ye ZC. Glutamate/ glutamine metabolism coupling between astrocytes and glioma cells: neuroprotection and inhibition of glioma growth. Biochem Biophys Res Commun 2014;450:295-9.
12. Meyer RP, Gehlhaus M, Knoth R, Volk B. Expression and function of cytochrome p450 in brain drug metabolism. Curr Drug Metab 2007;8:297-306.

13. Gonzalez-Perez O, Gutierrez-Fernandez F, Lopez-Virgen V, Collas-Aguilar J, Quinones-Hinojosa A, Garcia-Verdugo JM. Immunological regulation of neurogenic niches in the adult brain. Neuroscience 2012;226:270-81.

14. Lo Vasco VR, Fabrizi C, Panetta B, Fumagalli L, Cocco L. Expression pattern and sub-cellular distribution of phosphoinositide specific phospholipase $\mathrm{C}$ enzymes after treatment with U-73122 in rat astrocytoma cells. J Cell Biochem 2010;110:1005-12.

15. Rivera-Zengotita M, Yachnis AT. Gliosis versus glioma?: don't grade until you know. Adv Anat Pathol 2012;19:239-49.

16. Anderová M, Kubinová S, Mazel T, Chvátal A, Eliasson C, Pekny M, Sykova E. Effect of elevated $\mathrm{K}(+)$, hypotonic stress, and cortical spreading depression on astrocyte swelling in GFAP-deficient mice. Glia 2001;35:189-203

17. Jo S, Yarishkin O, Hwang YJ, Chun YE, Park M, Woo DH, Bae JY, Kim T, Lee J, Chun H, Park HJ, Lee da Y, Hong J, Kim HY, Oh SJ, Park SJ, Lee H, Yoon BE, Kim Y, Jeong Y, Shim I, Bae YC, Cho J, Kowall NW, Ryu H, Hwang E, Kim D, Lee CJ. GABA from reactive astrocytes impairs memory in mouse models of Alzheimer's disease. Nat Med 2014;20:886-96.

18. Tong CK, Alvarez-Buylla A. SnapShot: adult neurogenesis in the V-SVZ. Neuron 2014;81:220-220.e1.

19. Gonzalez-Perez O, Alvarez-Buylla A. Oligodendrogenesis in the subventricular zone and the role of epidermal growth factor. Brain Res Rev 2011;67:147-56.

20. Edgar N, Sibille E. A putative functional role for oligodendrocytes in mood regulation. Transl Psychiatry 2012;2:e109.

21. Seri B, García-Verdugo JM, McEwen BS, Alvarez-Buylla A Astrocytes give rise to new neurons in the adult mammalian hippocampus. J Neurosci 2001;21:7153-60.

22. Prochnow N, Abdulazim A, Kurtenbach S, Wildförster V, Dvoriantchikova G, Hanske J, Petrasch-Parwez E, Shestopalov VI, Dermietzel R, Manahan-Vaughan D, Zoidl G. Pannexin1 stabilizes synaptic plasticity and is needed for learning. PLoS One 2012; 7:e51767.

23. Menn B, Garcia-Verdugo JM, Yaschine C, Gonzalez-Perez O, Rowitch D, Alvarez-Buylla A. Origin of oligodendrocytes in the subventricular zone of the adult brain. J Neurosci 2006;26:7907-18.

24. Deng Y, Xie D, Fang M, Zhu G, Chen C, Zeng H, Lu J, Charanjit K. Astrocyte-derived proinflammatory cytokines induce hypomyelination in the periventricular white matter in the hypoxic neonatal brain. PLoS One 2014;9:e87420.

Cite this article as: Gonzalez-Perez O, Lopez-Virgen V, Quiñones-Hinojosa A. Astrocytes: everything but the glue. Neuroimmunol Neuroinflammation 2015;2(2):115-7

Source of Support: Nil. Conflict of Interest: No.

Received: 15-09-2014; Accepted: 10-11-2014 